\title{
Étude de l'influence des facteurs du milieu sur la croissance et la production du liège de la suberaie du Parc national de Theniet El Had (Tissemsilt, Algérie)
}

\section{Oumeldjilali NAGGAR ${ }^{1}$ \\ Rachid Tarik BOUHRAOUA ${ }^{1}$ \\ Enrique TORRES ${ }^{2}$ \\ Mohamed ZEDEK ${ }^{3}$}

\section{${ }^{1}$ Université de Tlemcen}

Département des ressources forestières Laboratoire de gestion conservatoire de l'eau, sols et forêts 22, rue Abi Ayed Abdelkrim, Fg Pasteur BP 119, 13000 Tlemcen Algérie

2 Universidad de Huelva Departamento de Ciencias Agroforestales Dr. Cantero Cuadrado, 6 21004 Huelva

Espagne

${ }^{3}$ Université Ibn Khaldoun Tiaret Faculté des sciences de la nature et de la vie

BP P 78, Zaâroura

14000 Tiaret

Algérie

\section{Auteur correspondant /} Corresponding author: Oumeldjilali Naggar naggaroumeldjilali@yahoo.fr

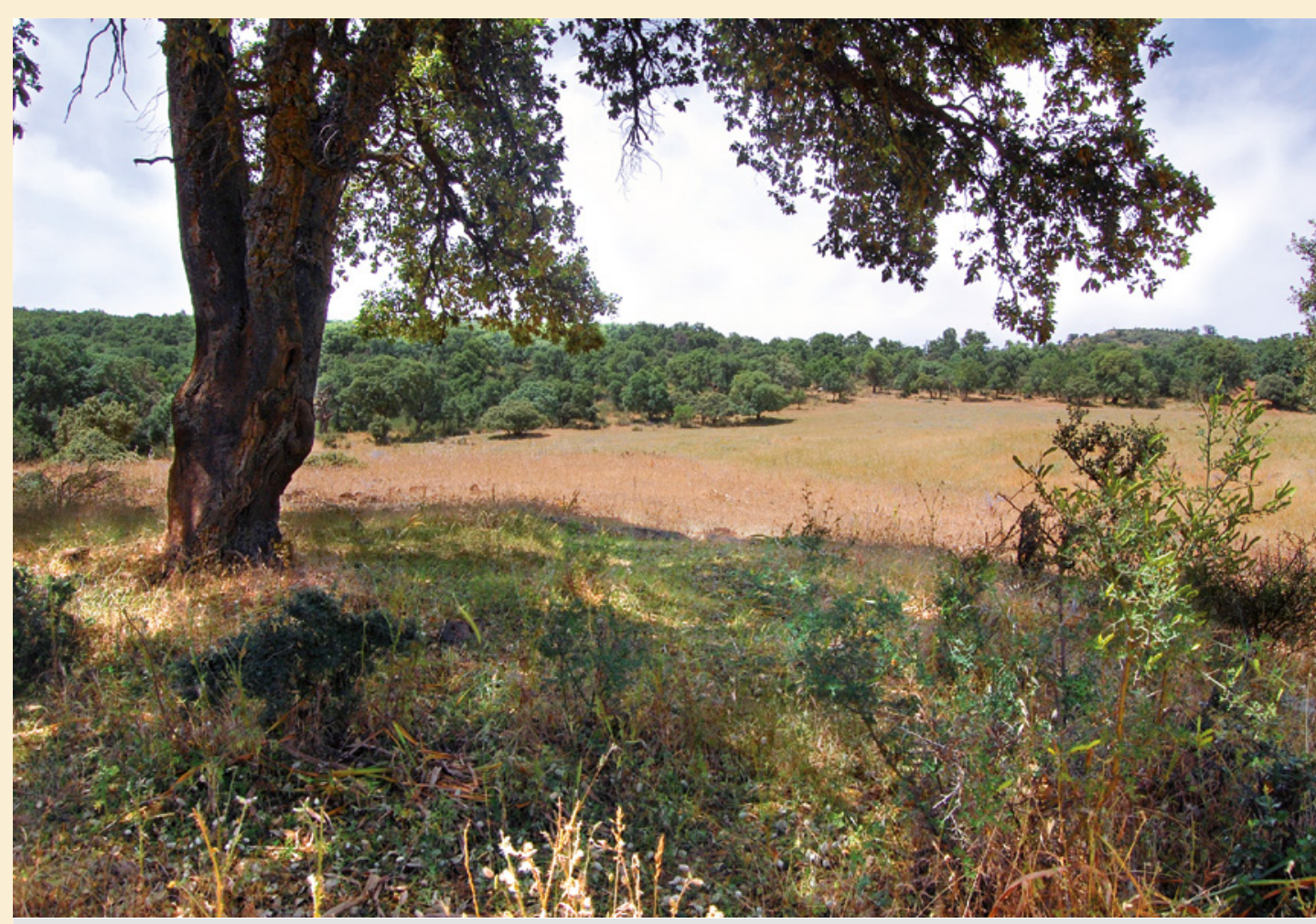

Photo 1.

Suberaie pure en basses altitudes (1 $292 \mathrm{~m}$ ).

Photo O. Naggar, 2013.

Doi : 10.19182/bft2019.342.a31793 - Droit d'auteur (c) 2019, Bois et Forêts des Tropiques @ Cirad - Date de soumission : 3 septembre 2018 ; date d'acceptation : 3 avril 2019 ; date de publication : 15 octobre 2019.

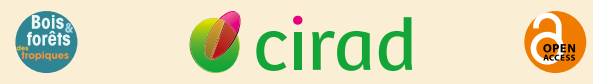

(c) $(1) \Theta$ 


\section{RÉSUMÉ}

\section{Étude de l'influence des facteurs du milieu sur la croissance et la production du liège de la suberaie du Parc national de Theniet El Had (Tissemsilt, Algérie)}

La variation des capacités de production de liège en relation avec les facteurs du milieu a été évaluée à partir de 53 placettes temporaires installées (763 arbres) dans la forêt montagneuse de chêne-liège du Parc national de Teniet El Had (Algérie). Trois paramètres de production sont retenus : l'épaisseur ( $\mathrm{mm} /$ an), le rendement d'un arbre moyen $(\mathrm{kg})$ et la productivité d'un peuplement ( $\mathrm{kg} /$ ha). Le présent travail montre l'effet des principaux facteurs du milieu sur l'épaisseur et la production de liège, identifié à partir de modèles linéaires généralisés. Les résultats ont montré que la croissance du liège est lente $(2,38 \mathrm{~mm} /$ an) et qu'elle est davantage affectée par des variables propres à l'arbre (état de santé, diamètre et hauteur d'écorçage) que par les sites (exposition). Cependant, le rendement est influencé par les conditions de croissance des arbres, exprimées par leur hauteur totale mais aussi par leur état de santé. À l'échelle des peuplements, deux qualités de sites sont proposées en fonction de la valeur de la hauteur dominante exprimée par le modèle : d'une part, les sites de bonne qualité (Hd > 10 m ; 2900 kg/ha) caractérisés par des arbres de bonne croissance en largeur et, d'autre part, les sites de mauvaise qualité avec une productivité réduite de plus de moitié ( $\mathrm{Hd}<10 \mathrm{~m}$; $1700 \mathrm{~kg} / \mathrm{ha}$ ). Ces résultats fournissent une orientation de gestion permettant au forestier de planifier la récolte de liège de sa forêt selon la rotation pratiquée et la qualité de la planche recherchée.

Mots-clés : chêne-liège, liège, facteurs de milieu, rendement, croissance, modèle, Algérie.

\section{ABSTRACT}

Study on the influence of environmental factors on tree growth and cork production in the cork oak forests of Theniet El Had National Park (Tissemsilt, Algeria)

Variations in cork production capacities according to environmental factors were assessed in 53 temporary survey plots (763 trees) in the montane cork oak forests of Teniet El Had National Park (Algeria). Three production parameters were assessed: thickness ( $\mathrm{mm} /$ year), yield $(\mathrm{kg})$ from an average tree and productivity $(\mathrm{kg} / \mathrm{ha})$ of a stand. Our study presents the effects of the main environmental factors on cork thickness and production, as identified from generalised linear models. The results show that cork grows slowly $(2.38 \mathrm{~mm} /$ year) and that growth rates are affected more by tree-specific variables (state of health, barking diameter and height) than by site factors (exposure). On the other hand, yields are influenced by the conditions of tree growth, as expressed by their total height but also by their state of health. In terms of stand quality, the sites fall into two types depending on the dominant height expressed by the model: (i) high-quality sites ( $\mathrm{Hd}>10 \mathrm{~m}$; $2,900 \mathrm{~kg} / \mathrm{ha}$ ) characterised by trees with high growth rates in diameter, and (ii) poor quality sites where productivity is less than half of the former $(\mathrm{Hd}<10 \mathrm{~m}$; $1,700 \mathrm{~kg} / \mathrm{ha}$ ). These results provide management guidelines to help foresters plan cork harvests from their forests according to rotations and to the desired cork quality.

Keywords: cork oak, cork, environmental factors, yield, growth, model, Algeria.

\section{Estudio de la influencia de los factores del medio en el crecimiento y la producción de corcho del alcornoque del Parque Nacional de Theniet El Had (Tissemsilt, Argelia)}

Se evaluó la variación de la capacidad de producción de corcho en relación con los factores del medio a partir de 53 parcelas de ensayo temporales instaladas (763 árboles) en el bosque montañoso de alcornoques del Parque Nacional de Teniet El Had (Argelia). Se escogieron tres parámetros de producción: grosor (mm/año), rendimiento de un árbol medio $(\mathrm{kg})$ y productividad de una población ( $\mathrm{kg} / \mathrm{ha}$ ). Este trabajo presenta el efecto de los principales factores del medio en el grosor y la producción del corcho, a partir de modelos lineales generalizados. Los resultados mostraron que el crecimiento del corcho es lento (2,38 mm/año) y que está más intensamente afectado por variables propias del árbol (estado de salud, diámetro y altura de raspado) que por su situación (exposición). En cambio, el rendimiento está influido por las condiciones de crecimiento de los árboles, evidenciadas por la altura total, aunque también por su estado de salud. A escala de las masas forestales, se propusieron dos tipos de calidad de los lugares en función del valor de la altura dominante expresada por el modelo: (i) los espacios de buena calidad ( $\mathrm{Hd}>10 \mathrm{~m} ; 2900 \mathrm{~kg} / \mathrm{ha}$ ) caracterizados por árboles de buen crecimiento en anchura, y (ii) los espacios de mala calidad con una productividad reducida en más de la mitad ( $\mathrm{Hd}<10 \mathrm{~m} ; 1700 \mathrm{~kg} / \mathrm{ha}$ ). Estos resultados ofrecen una orientación de gestión que permite al forestal planificar la recogida de corcho de su bosque según la rotación practicada y la calidad de la plancha buscada.

Palabras clave: alcornoque, corcho, factores del medio, rendimiento, crecimiento, modelo, Argelia. 


\section{Introduction}

Le chêne-liège (Quercus suber L.) est considéré depuis plusieurs siècles comme une ressource naturelle prestigieuse dans les régions forestières ouest-méditerranéennes. Il est réparti entre sept pays dont quatre du sud de l'Europe (Portugal, Espagne, France et Italie) et trois du nord de l'Afrique (Algérie, Tunisie et Maroc). En Algérie, ses forêts constituent des paysages typiques, aux intérêts multiples, de nature sociale, économique et environnementale. Mais la grande valeur économique de ces forêts réside dans la production de liège, produit de base d'une économie forestière propre à la région. Le liège est le sixième produit forestier non ligneux exporté au monde ; il génère environ deux milliards de dollars de chiffre d'affaires annuel dont $70 \%$ proviennent de la fabrication de bouchons naturels (FAO, 2013).

Pour assurer leur pérennité et la régularité des récoltes sans affecter leur vitalité, les peuplements de chêne-liège sont soumis à deux types de traitements complémentaires, la sylviculture et la subériculture (Lamey, 1893 ; Natividade, 1956). Le premier assure la reconstitution de la suberaie par des soins culturaux et des aménagements. Le second vise à produire, en quantité et de manière soutenable, un liège de bonne qualité selon trois paramètres essentiels : l'épaisseur, la porosité et les défauts du liège (Ferreira et al., 2000 ; Pereira, 2007 ; Ghalem et al., 2018). La combinaison de ces paramètres dégage sept classes commerciales de qualité de liège, allant de la bonne qualité, destinée à la fabrication de bouchons naturels d'une seule pièce, au liège de rebut, orienté vers l'industrie de trituration (Pereira et al., 1996 ; Sánchez-González et al., 2005). De nombreuses recherches ont été menées dans plusieurs pays sur les capacités productives des suberaies en relation avec les facteurs du milieu (Montero et al., 1996 ; Torres et al., 1997 ; Costa et al., 2002 ; Paulo et al., 2015 ; Costa et al., 2016 ; Mendes et al., 2016 ; Ghalem et al., 2018). Les résultats obtenus ont permis l'optimisation de la croissance et ont été par conséquent le prélude à plusieurs modèles de prédiction des capacités de production de liège (Cuevas-Gozalo, 1995 ; Sánchez-González et al., 2005 ; Vázquez-Piqué et Pereira, 2005 ; Paulo et al., 2015, 2016 ; Paulo et Tomé, 2017).

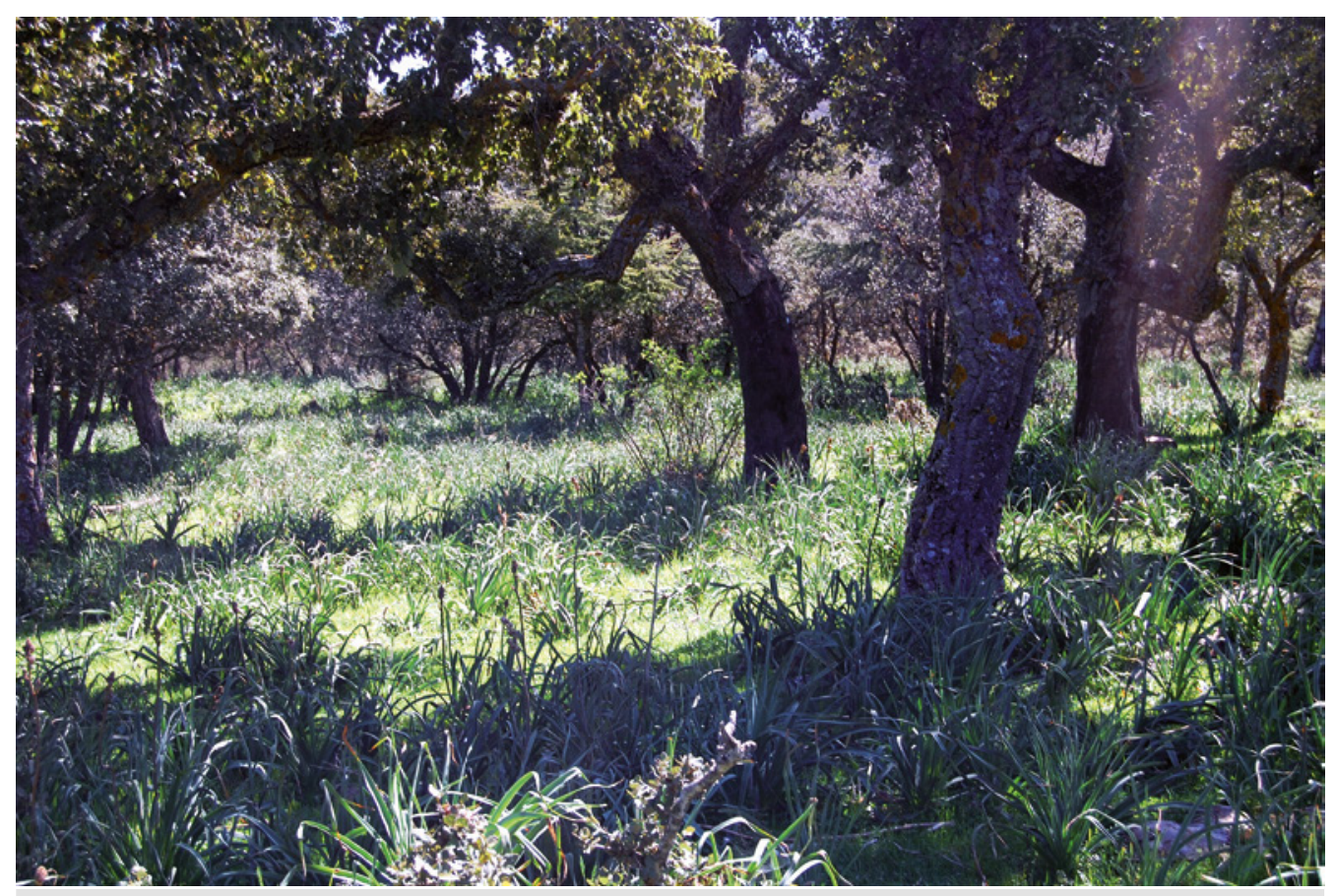

Photo 2.

Suberaie pure à une altitude moyenne (1450 m).

Photo O. Naggar, 2013.
En Algérie, les forêts à Quercus suber couvraient auparavant une superficie de près de 460000 ha (Lamey, 1893) ; elles sont concentrées principalement dans la région nord-est du pays (Boudy, 1955). Cependant, le premier inventaire forestier national de 1983/1984 n'a révélé que 230000 hectares productifs (Zine, 1992), ce qui représente une perte de près de moitié de la surface. La production moyenne de liège a chuté de $62 \%$ en passant de 31300 tonnes par an dans les années quarante (Natividade, 1956) à 12000 tonnes par an depuis la relance de la filière liège en 1998 (Dehane et al., 2013a). La réduction des superficies productives du liège est due essentiellement aux incendies récurrents qui ravagent en moyenne près de 7000 ha par an de suberaies (DGF, 2013), mais aussi aux contraintes de reboisement (Bouhraoua et al., 2014) et à une politique de gestion insuffisamment efficace (Dehane et al., 2013a). En dépit de leur importance, les études relatives aux estimations de la production de liège avant la récolte, en relation avec les caractéristiques physiques et sylvicoles des peuplements, sont rares. Or, ce type de recherche s'impose à l'heure actuelle pour prédire l'avenir de la filière liège en Algérie.

Le présent travail avait pour objectif de déterminer la rotation de la production du liège et d'identifier les facteurs stationnels et de peuplement influençant les capacités productives d'une forêt de chêne-liège relevant du Parc national de Theniet El Had. Dans cette zone, la forêt de chêne-liège était constituée de beaux peuplements à croissance lente offrant un liège d'excellente qualité (Boudy, 1955). Mais elle a été abandonnée depuis plusieurs décennies et les informations relatives à sa gestion forestière restent rares et fragmentaires. 


\section{Méthodes}

\section{Zone d'étude}

La zone d'étude est située sur le versant sud du Parc national de Theniet El Had qui fait partie du massif forestier d'Ouarsenis (Tissemsilt) (figure 1). Elle repose sur des grès medjaniens (Boudy, 1955). Les sols sont profonds à superficiels par endroits, de texture argilo-sablonneuse (PNTH, 2006). L'altitude varie de 1150 à $1600 \mathrm{~m}$ et la pente est généralement abrupte ( $25^{\circ}$ en moyenne). Le climat est méditerranéen avec des températures annuelles moyennes de $12{ }^{\circ} \mathrm{C}$, variant de -1 à $30^{\circ} \mathrm{C}$. Les précipitations moyennes atteignent $760 \mathrm{~mm} / \mathrm{an}$ (PNTH, 2006), ce qui soumet la zone d'étude à un bioclimat subhumide à hiver froid, selon la classification d'Emberger (Emberger, 1955). Sur une superficie de près de 700 ha, le chêne-liège est à l'état pur ou en mélange en proportions variées avec le cèdre de l'Atlas (Cedrus atlantica) ou avec d'autres chênes (Quercus ilex et Q. canariensis).

\section{Échantillonnage}

L'étagement altitudinal des divers peuplements forestiers, leur répartition discontinue et leur hétérogénéité structurale nous ont imposé le principe d'échantillonnage dirigé en se basant sur la localisation du chêne-liège qui constitue visiblement un peuplement pur (figure 1). L'unité d'échantillonnage retenue est un cercle de surface fixe de 5 ares (un rayon de 12,62 m) contenant au moins 10 tiges (Rondeux, 1999). Au total, 53 placettes ont été installées et inventoriées durant les années 2012 et 2013.

\section{Variables placettes}

Chaque placette a été délimitée par la mire de Pardé et le dendromètre Blume-Leiss (Rondeux, 1999) et a fait l'objet d'une description détaillée des conditions du milieu dans lesquelles évoluent les peuplements inventoriés. L'altitude (Alt) a été notée en trois classes (inférieure, moyenne et supérieure) selon un intervalle de $100 \mathrm{~m}$ d'altitude. L'exposition (Expo) relevée à l'aide d'une boussole forestière a été enregistrée en trois modalités selon la direction dominante des pluies ou des pentes. De même, la pente du terrain ( $P$ ) a été mesurée par le Blume-Leiss et les positions topographiques (Topo) ont été décrites selon quatre niveaux (Masson, 2005) : Topo 1 (pertes latérales d'eau supérieures aux apports), Topo 2 (apports nuls ou égaux aux pertes d'eau par drainage), Topo 3 (situation favorable avec une circulation lente de l'eau) et Topo 4 (situation hydrique exceptionnellement favorable). Enfin, les conditions pédologiques ont

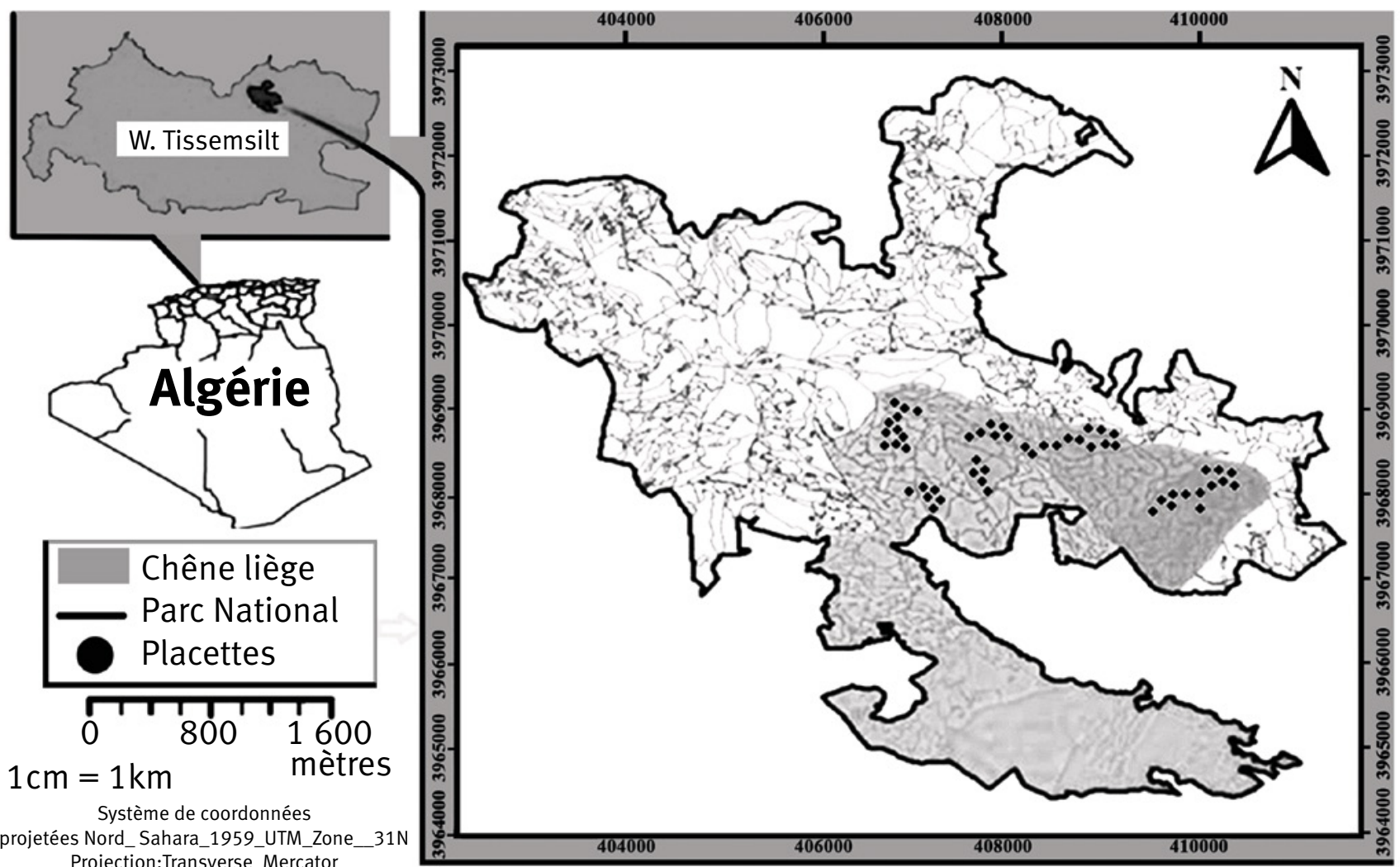


été approchées par la variable profondeur maximale du sol (PS), obtenue par des tests à la tarière. Elle correspond à la moyenne des profondeurs classées en trois catégories (Richard, 1988) : superficiel, assez profond et profond.

Les peuplements ont été décrits selon trois critères : circonférence et hauteur dominante, variables liées à la fertilité stationnelle (Sánchez-González et al., 2010), la hauteur dominante correspondant à la moyenne arithmétique des 100 plus gros bois à l'hectare (Rondeux, 1999); la densité (Nt) qui reflète le degré de compétition entre les arbres (Rondeux, 1999) ; l'état de santé des peuplements. Ce dernier a été évalué par l'indice de santé (IS), calculé à partir du déficit foliaire (Df) estimé directement sur les arbres inventoriés. Cet indice a déjà été utilisé pour le chêne-liège et déterminé à partir de la formule suivante (Bouhraoua et Villemant, 2005) :

$$
I S=\sum_{n=1}^{4} \mathrm{ni} \times \mathrm{pi} / \mathrm{N}
$$

avec : $n$, nombre d'arbres de chaque classe de déficit foliaire (Df) ; pi, poids de la classe $i$ de déficit foliaire, valant 1 si $i=1$ (classe d'arbres sains), 2 si $i=2$ (arbre moyennement défolié), 3 si $i=3$ (arbre fortement défolié), 4 si $i=4$ (arbre mort ou sec) ; N, effectif total d'arbres observés dans la placette.

Selon les valeurs de l'indice, le peuplement est considéré comme sain $\left(\mathrm{IS}_{1}<1,6\right)$, affaibli $\left(1,6 \leq \mathrm{IS}_{1} \leq 2,0\right)$, ou en dépérissement $\left(I S_{1}>2,0\right)$.

\section{Variables arbres}

Au total, 763 arbres présents dans les placettes ont été pris en considération dans l'inventaire. Ils ont fait l'objet des mesures dendrométriques suivantes : hauteur totale $(\mathrm{Ht})$, circonférence sur liège à $1,30 \mathrm{~m}$ du sol (C) et hauteur du fût ( $\mathrm{Hf}$ ). La vitalité des arbres portait essentiellement sur l'état de leur cime estimé par le symptôme « déficit foliaire » couramment employé dans le réseau européen de surveillance de l'état sanitaire des forêts (DSF, 1991). Les différentes notes ont été converties en quatre classes d'état de santé : (1) pas de déficit foliaire visible ( $25 \%$ : houppier opaque avec ramification dense), (2) déficit foliaire moyen (30-60\%), (3) déficit foliaire fort ( $\geq 65 \%$ ) et (4) arbre mort au houppier complètement desséché.

\section{Données arbres exploitées}

Les arbres inventoriés ont été répartis en deux catégories : les sujets non exploités (NE) recouverts encore de liège naturel et les sujets exploités portant le liège de reproduction $(n=379)$. Cette dernière catégorie d'arbres $(50 \%$ de l'effectif total) a fait l'objet de mesures de l'épaisseur du liège $(E)$, qui est la moyenne des mesures prises aux quatre points cardinaux, et de la hauteur d'écorçage (HE) lors de la dernière récolte, encore visible.

\section{Données des échantillons et détermination du cycle de production du liège}

L'estimation de la quantité de liège fournie par les arbres et les peuplements en fin de cycle de production commence par la détermination de la durée de ce cycle de production fournissant une épaisseur commerciale adaptée à la fabrication de bouchons. Pour cela, nous avons retenu une épaisseur de référence de $30 \mathrm{~mm}$ sur écorce (González-Adrados et al., 2000 ; Paulo et al., 2011). L'échantillonnage a consisté à extraire, à partir de deux arbres de chaque placette (exceptionnellement trois arbres), des plaques de liège de $10 \mathrm{~cm} \times 10 \mathrm{~cm}$ à $1,30 \mathrm{~m}$ du sol. Au total, 125 plaques de liège ont été prélevées et bouillies à $100{ }^{\circ} \mathrm{C}$ pendant une heure (Ferreira et al., 2000). Plusieurs paramètres ont été mesurés : épaisseur sur croûte sur les quatre côtés $(\mathrm{m})$, surface $\left(\mathrm{m}^{2}\right)$ et poids sec $(\mathrm{kg})$. Ceux-ci ont permis le calcul du poids du liège produit par unité de surface d'écorçage $\left(\mathrm{kg} / \mathrm{m}^{2}\right)$ et de la masse volumique $(\mathrm{kg} /$ $\mathrm{m}^{3}$ ). L'âge de chaque échantillon de liège a été estimé par le dénombrement des cernes subéreux au moyen d'un outil de dendrochronologie, Lintab5. La largeur de ces cernes a été mesurée à deux endroits sur chacune des deux sections transversales. Seuls les résultats des accroissements annuels complets ont été pris en compte, en excluant les deux accroissements incomplets (le premier et le dernier) correspondant aux années de récolte, auxquels s'ajoute l'épaisseur de la croûte. Si un échantillon offre $n$ anneaux distincts, il est donc âgé de $(n-1)$ années (Saccardy, 1937 ;

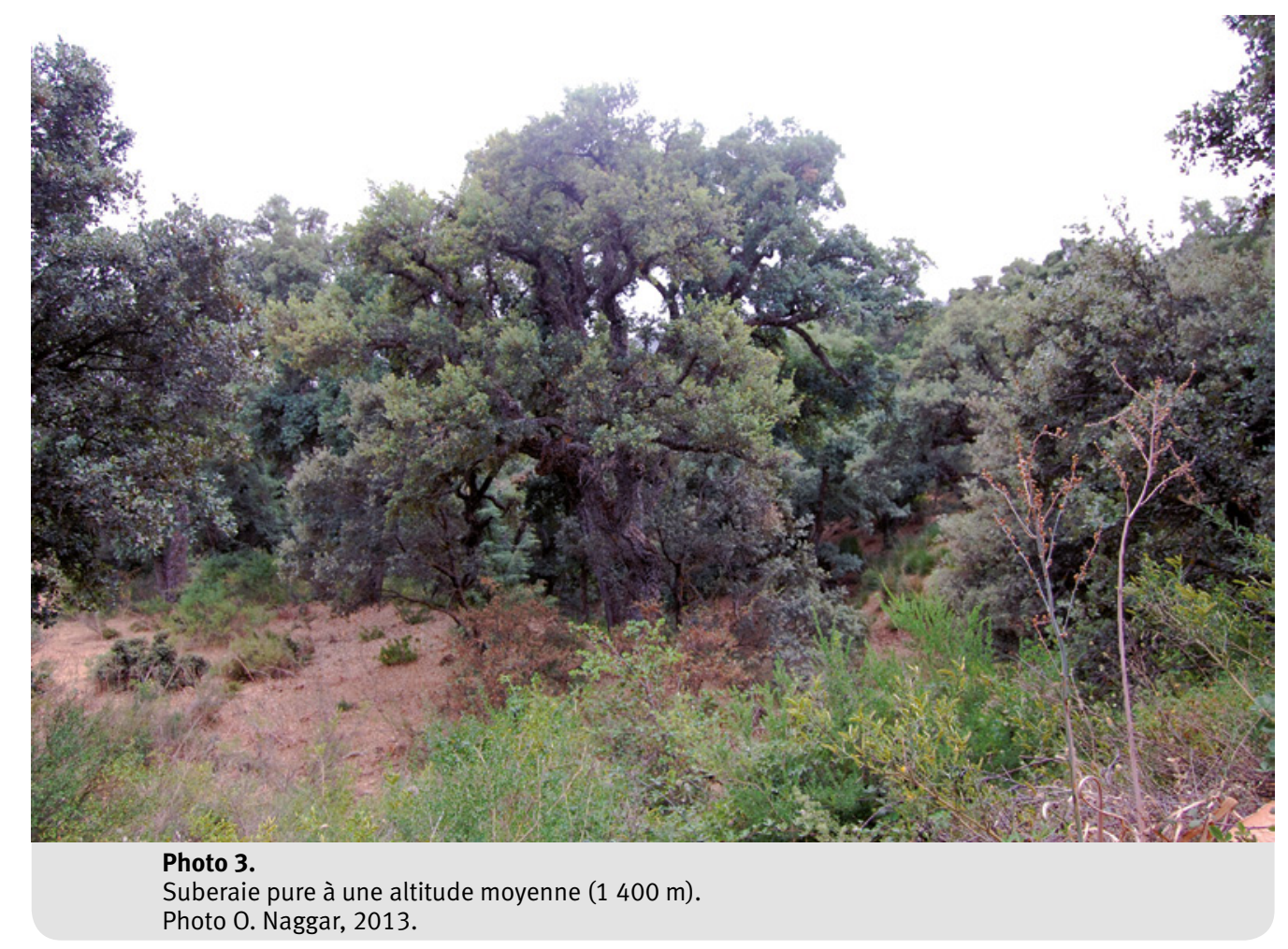


Natividade, 1956). L'ensemble des échantillons a été classé en catégories de liège selon l'épaisseur totale atteinte en fin de cycle de production (Pereira, 2007) : mince (< $27 \mathrm{~mm})$, juste (27-32 mm), régulier (32-40 mm) et épais (> $40 \mathrm{~mm})$.

\section{Rendement des arbres et production des peuplements}

La production de liège fournie par un arbre moyen sur pied et celle produite par un peuplement ont été déterminées à partir des formules simples anciennement proposées par Lamey (1893) et Saccardy (1937) pour le liège algérien et par d'autres auteurs pour le liège ibérique (Montero et al.,

Tableau I.

Fréquences relatives ( $\mathrm{Fr}$ ) de chaque modalité de variables qualitatives des placettes $(n=53)$ et des arbres échantillonnés $(n=634)$.

\begin{tabular}{|l|c|c|c|c|} 
Variables & Code & Modalité 1 & Modalité 2 & Modalité 3 \\
\hline Exposition & Expo $^{1}$ & $0,26(0,48)$ & $0,53(0,18)$ & $0,23(0,35)$ \\
\hline Pente $\left(^{\circ}\right)$ & $\mathrm{P}^{2}$ & $0,26(0,32)$ & $0,53(0,49)$ & $0,21(0,19)$ \\
\hline Position topographique & Topo $^{3}$ & $0,43(0,48)$ & $0,19(0,18)$ & $0,38(0,35)$ \\
\hline Altitude $(\mathrm{m})$ & $\mathrm{Alt}^{4}$ & $0,21(0,24)$ & $0,68(0,66)$ & $0,11(0,10)$ \\
\hline Profondeur du sol $(\mathrm{cm})$ & $\mathrm{PS}^{5}$ & $0,08(0,09)$ & $0,40(0,39)$ & $0,53(0,52)$ \\
\hline Densité totale (tiges/ha) & $\mathrm{Nt}^{6}$ & $0,17(0,12)$ & $0,51(0,42)$ & $0,32(0,46)$ \\
\hline
\end{tabular}

${ }^{1}$ Exposition (Expo) en modalités : 1 (SW : favorable) ; 2 (S : peu favorable) ; 3 (SE : défavorable). ${ }^{2}$ Pente $(P)$ en modalités : 1 (plat : $\left\langle 9^{\circ}\right) ; 2$ (moyen : $10-20^{\circ}$ ); 3 (abrupt : > $20^{\circ}$ ). ${ }^{3}$ Position topographique (Topo) en modalités : 1 (très favorable : Topo4) ; 2 (favorable : Topo3) ; 3 (défavorable : Topo1-2). ${ }^{4}$ Altitude (Alt) en modalités : 1 (1 290-1 $390 \mathrm{~m})$; 2 (1 390-1 $490 \mathrm{~m})$; 3 (> $1490 \mathrm{~m}) .{ }^{5}$ Profondeur du sol (PS) en modalités : 1 (profond : > $60 \mathrm{~cm}$ ) ; 2 (assez profond : 30-60 cm) ; 3 (superficielle : $<30 \mathrm{~cm}$ ). ${ }^{6}$ Densité totale du peuplement (Nt) en modalités : 1 (claire : $<200$ tiges/ha) ; 2 (moyenne : 200-300 tiges/ha) ; 3 (dense : > 300 tiges/ha).

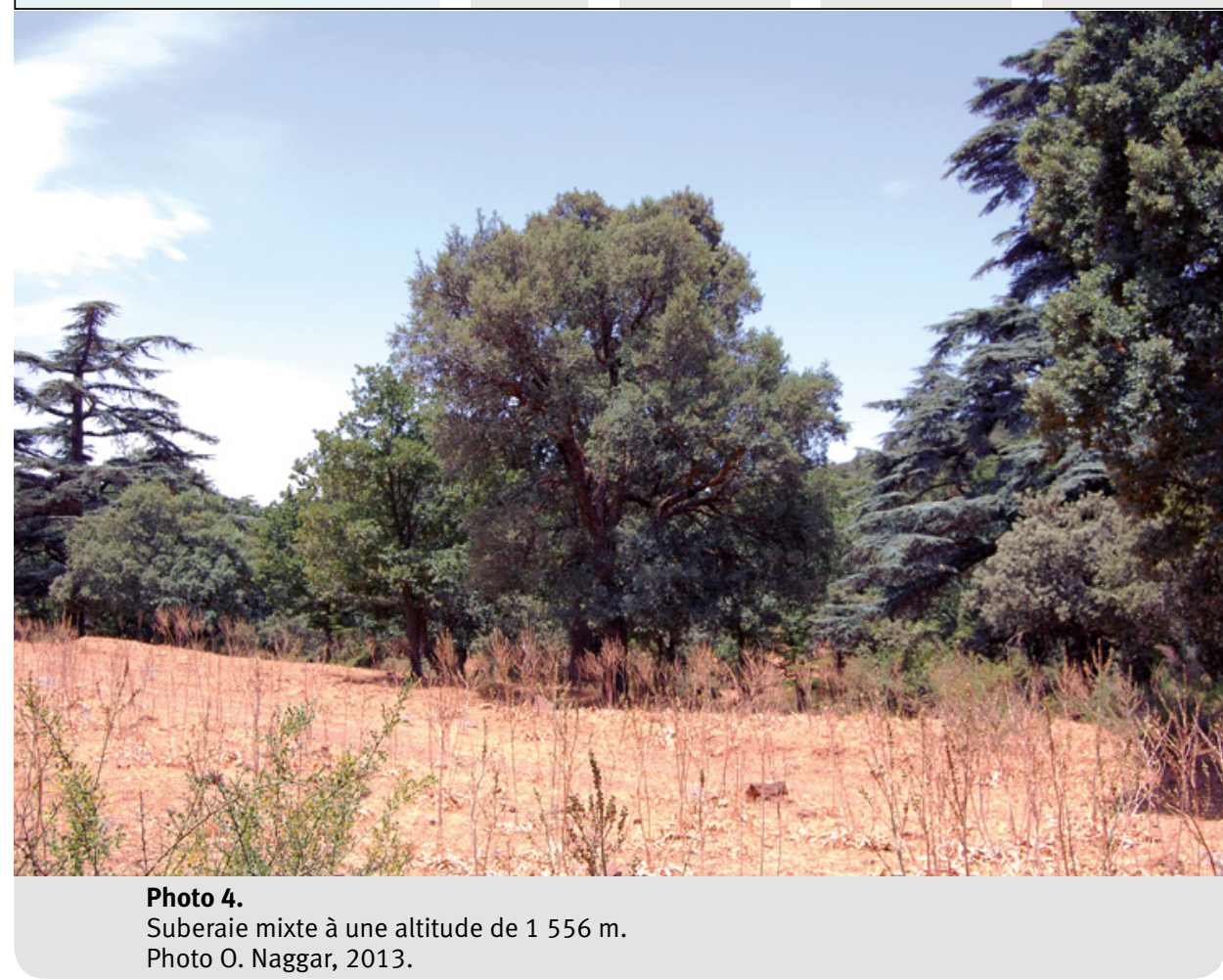

avec : $C$, circonférence sur liège $(\mathrm{m})$; $E$, épaisseur du liège $(\mathrm{m}) ; H E$, hauteur d'écorçage (m).

Les potentialités productives du liège liées aux conditions du milieu ont été déterminées en recourant à deux paramètres : le rendement des arbres $\left(R_{n}\right)$ et la production du peuplement $\left(P_{n}\right)^{n}$.

Le rendement $(\mathrm{kg})$ de liège fourni par un arbre sur une rotation de $n$ années est tiré de la formule suivante :

$$
R n=1,5(C-2 \pi \cdot E n)^{\wedge} 2 . \rho
$$

où $C$ et $E$ sont les variables indiquées précédemment, $E n$ est l'épaisseur moyenne cumulée du liège (m) atteinte en $n$ années d'accroissement complet, $\rho$ est la masse volumique du liège $\left(\mathrm{kg} / \mathrm{m}^{3}\right)$; le coefficient d'écorçage minimal est de 1,5 (Boudy, 1950).

La production $(\mathrm{kg} / \mathrm{ha})$ du peuplement est estimée sur la base du rendement moyen des arbres de la placette $\left(R_{p}\right)$ et de la densité du peuplement $(\mathrm{Nt})$.

\section{Analyses statistiques des données}

Pour mettre en évidence les variables du milieu influençant la croissance du liège, nous avons utilisé la régression logistique binomiale $(\mathrm{glm})$. Pour cela, nous avons analysé comme variable dépendante l'épaisseur cumulée du liège atteinte en fin de cycle de production de 125 plaques échantillonnées. Dans une première étape, nous avons séparé ces échantillons en deux groupes par codage binaire selon l'utilisation industrielle de la planche du liège : 1 pour les plaques bouchonnables d'une épaisseur supérieure à 30 mm 
sur croûte, et 0 pour les plaques non bouchonnables d'une épaisseur inférieure à $30 \mathrm{~mm}$. Les mesures de toutes les plaques ont été majorées de $4 \mathrm{~mm}$, valeur correspondant à la croûte et aux deux demi-accroissements.

Dans une seconde étape, nous avons soumis à l'analyse les différentes variables indépendantes (explicatives) recueillies au niveau des arbres et des placettes (tableau II).

Pour tester l'influence des variables sur le rendement potentiel en liège des arbres atteint en fin de cycle de production ( $R_{n}$ : variable réponse), nous n'avons pris en considération que les arbres ayant une dimension exploitable $\left(\emptyset_{\text {sous écorce }}>14 \mathrm{~cm}\right)$ indépendamment qu'ils soient déjà récoltés ou non. Pour cela, nous avons soumis le rendement individuel en liège des arbres $(n=634)$ aux différentes variables explicatives consignées dans les tableaux I et II tout en évitant d'inclure celles qui sont redondantes ou fortement corrélées, telle la grosseur du tronc des arbres (Paulo et Tomé, 2010). Enfin, à l'échelle des placettes, nous avons soumis à l'analyse la réponse " production du peuplement » aux différentes variables explicatives.

Toutes les analyses des données ont été réalisées en utilisant les fonctions $\mathrm{glm}$ et $\mathrm{lm}$ du module stats à l'aide du logiciel R, version 3.5.1 (R Core Team, 2018). Pour chaque type de réponse (quantitative), nous avons commencé avec un modèle en incluant toutes les variables explicatives qui pourraient présenter une interprétation ou un sens biologique ou sylvicole. Pour vérifier si la sélection du modèle obtenu pourrait être améliorée, nous avons supprimé, à chaque étape, les variables qui n'ont pas un effet signifi- catif, jusqu'à ce que toutes les variables restantes dans le modèle soient significatives $(P<0,05)$. Pour chaque formulation d'un modèle, nous avons réalisé un diagnostic sur la multi-colinéarité entre les régresseurs des prédicteurs en utilisant la fonction Imcdiag du module mctest (Imdadullah et al., 2016). Plusieurs indices alternatifs ont été testés avant de sélectionner le modèle le plus performant. Il s'agit du critère d'information Akaike (AIC) et Bayésien (BIC) ; plus la valeur de ces critères est faible et plus le modèle indique une grande performance. En plus de ces tests, nous avons calculé la corrélation entre les variables explicatives (coefficient de corrélation de Pearson) et la corrélation multiple (R). Dans le cas où deux variables explicatives sont linéairement dépendantes, nous avons choisi dans le modèle celle qui a plus de sens.

\section{Résultats}

\section{Description du terrain et structure du peuplement}

L'analyse descriptive des différentes variables mesurées à deux niveaux (arbre et placette) est résumée dans le tableau I pour les variables qualitatives, et dans le tableau II pour les variables quantitatives. Ces tableaux montrent une forte variabilité pour la majorité des variables quantitatives (CV > $30 \%$ ). En effet, les placettes (peuplements) sont exposées davantage vers le sud-ouest (Fréquence relative : $48 \%$ ) que vers les autres orientations, avec une pente moyenne dominante de $12^{\circ}$ (49\% des placettes). La position

Tableau II.

Statistiques descriptives des variables quantitatives recueillies au niveau des placettes, des arbres individuels et des arbres servant à l'extraction du liège.

\begin{tabular}{|c|c|c|c|c|c|c|c|c|}
\hline $\begin{array}{l}\text { Niveau de } \\
\text { mesure }\end{array}$ & Variables & Code & Effectif & Moyenne & Minimum & Maximum & $\begin{array}{l}\text { Écart- } \\
\text { type }\end{array}$ & $\begin{array}{l}\text { Coefficient } \\
\text { de variation } \\
\text { (\%) }\end{array}$ \\
\hline \multirow[t]{5}{*}{ Placettes } & Hauteur dominante (m) & $\mathrm{Hd}$ & 53 & 8,71 & 4,3 & 15,35 & 2,29 & 26,29 \\
\hline & Indice de santé & IS & 53 & 1,58 & 1 & 2,58 & 0,34 & 22,11 \\
\hline & Surface terrière (m²/ha) & G & 53 & 27,21 & 4,78 & 60,7 & 14,11 & 51,85 \\
\hline & Densité totale (tiges/ha) & $\mathrm{Nt}$ & 53 & 277,07 & 140 & 780 & 104,40 & 37,68 \\
\hline & Densité exploitable (tiges/ha) & $\mathrm{NE}$ & 53 & 259,25 & 140 & 760 & 102,20 & 39,42 \\
\hline \multirow[t]{2}{*}{ Total arbres } & Circonférence à 1,30 m sous écorce (m) & $\mathrm{C}$ & 763 & 1.07 & 0,20 & 3,69 & 0,53 & 55.45 \\
\hline & Hauteur totale $(\mathrm{m})$ & $\mathrm{Ht}$ & 763 & 6,93 & 2,25 & 21,50 & 2,53 & 36,00 \\
\hline \multirow{5}{*}{$\begin{array}{l}\text { Arbres } \\
\text { exploitables }\end{array}$} & Circonférence à 1,30 m sur écorce (m) & $\mathrm{C}$ & 634 & 1,18 & 0,50 & 3,69 & 0,50 & 42,27 \\
\hline & Diamètre sous écorce (m) & $\mathrm{D}$ & 634 & 0,31 & 0,14 & 1,12 & 0,15 & 49,30 \\
\hline & Hauteur totale $(\mathrm{m})$ & $\mathrm{Ht}$ & 634 & 7,38 & 2,42 & 21,50 & 2,42 & 32,81 \\
\hline & Hauteur de fût (m) & $\mathrm{Hf}$ & 634 & 2,78 & 0,50 & 7,00 & 1,00 & 35,85 \\
\hline & Déficit foliaire (\%) & Df & 634 & 35,85 & 5 & 100 & 54,78 & 49,30 \\
\hline \multirow{7}{*}{$\begin{array}{l}\text { Arbres } \\
\text { exploités } \\
\text { Échantillons } \\
\text { de liège }\end{array}$} & Hauteur d’écorçage (m) & $\mathrm{HE}$ & 379 & 1,76 & 1,30 & 2,80 & 0,25 & 14,20 \\
\hline & Épaisseur du liège sur croûte (mm) & $E$ & 379 & 31,55 & 9,00 & 73,00 & 0,93 & 29,48 \\
\hline & Diamètre des arbres $(\mathrm{m})$ & Dc & 125 & 0,38 & 0,15 & 1,09 & 0,15 & 36,82 \\
\hline & Déficit foliaire des arbres (\%) & Dfc & 125 & 28.65 & 5 & 75 & 14.81 & 51.71 \\
\hline & Âge du liège (ans) & $A_{c}$ & 125 & 16,4 & 4 & 43 & 7,42 & 45,24 \\
\hline & Hauteur d’écorçage (m) & $\mathrm{HE}_{\mathrm{c}}$ & 125 & 1.17 & 1.30 & 2.80 & 0.28 & 16,87 \\
\hline & Épaisseur du liège sur croûte (mm) & $E_{c}^{c}$ & 125 & 35,67 & 10,15 & 64,18 & 11,05 & 30,98 \\
\hline
\end{tabular}




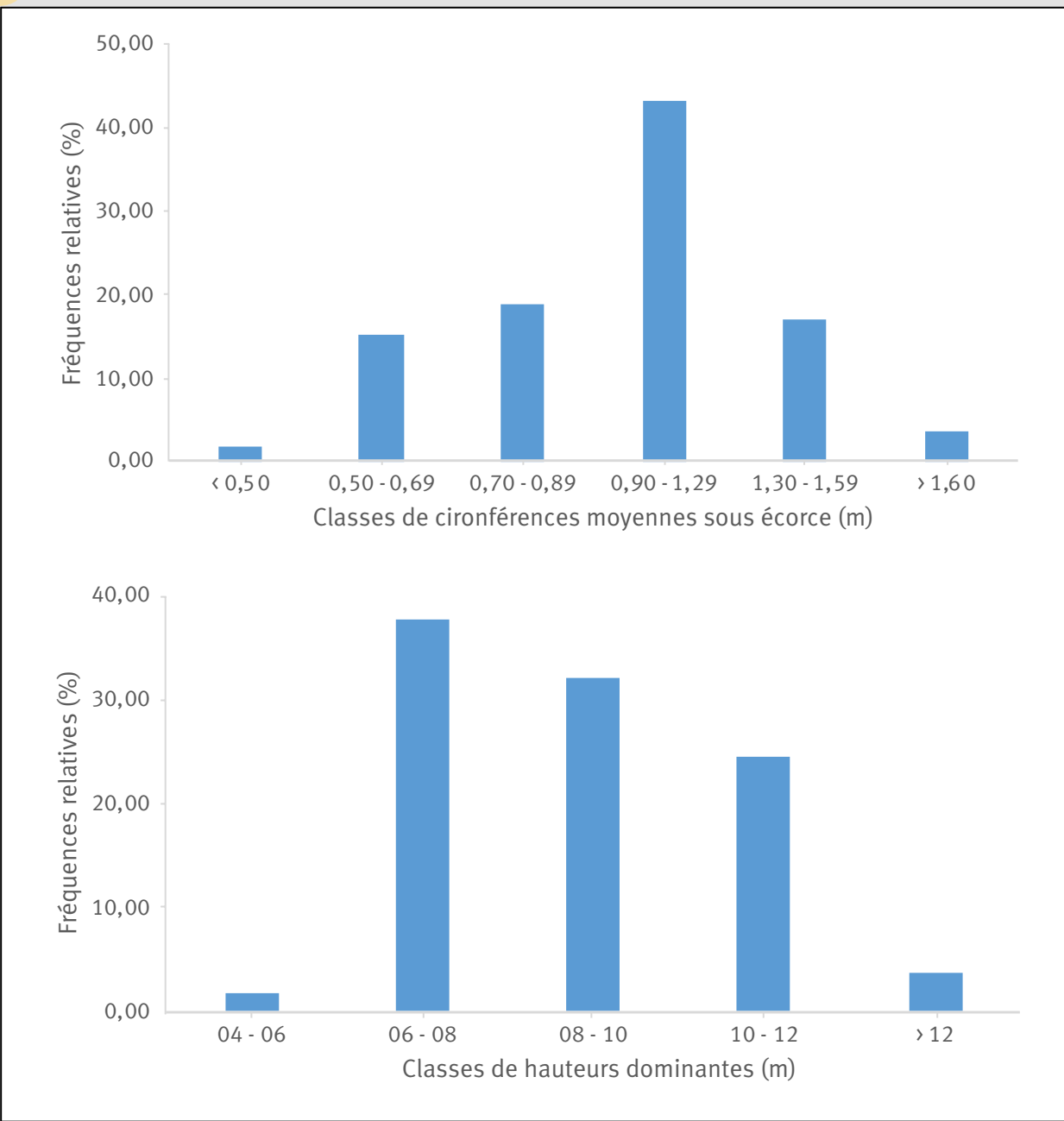

Figure 2.

Distribution par classe : (a) circonférences moyennes sous écorce et (b) hauteurs dominantes moyennes des 53 placettes d'étude.

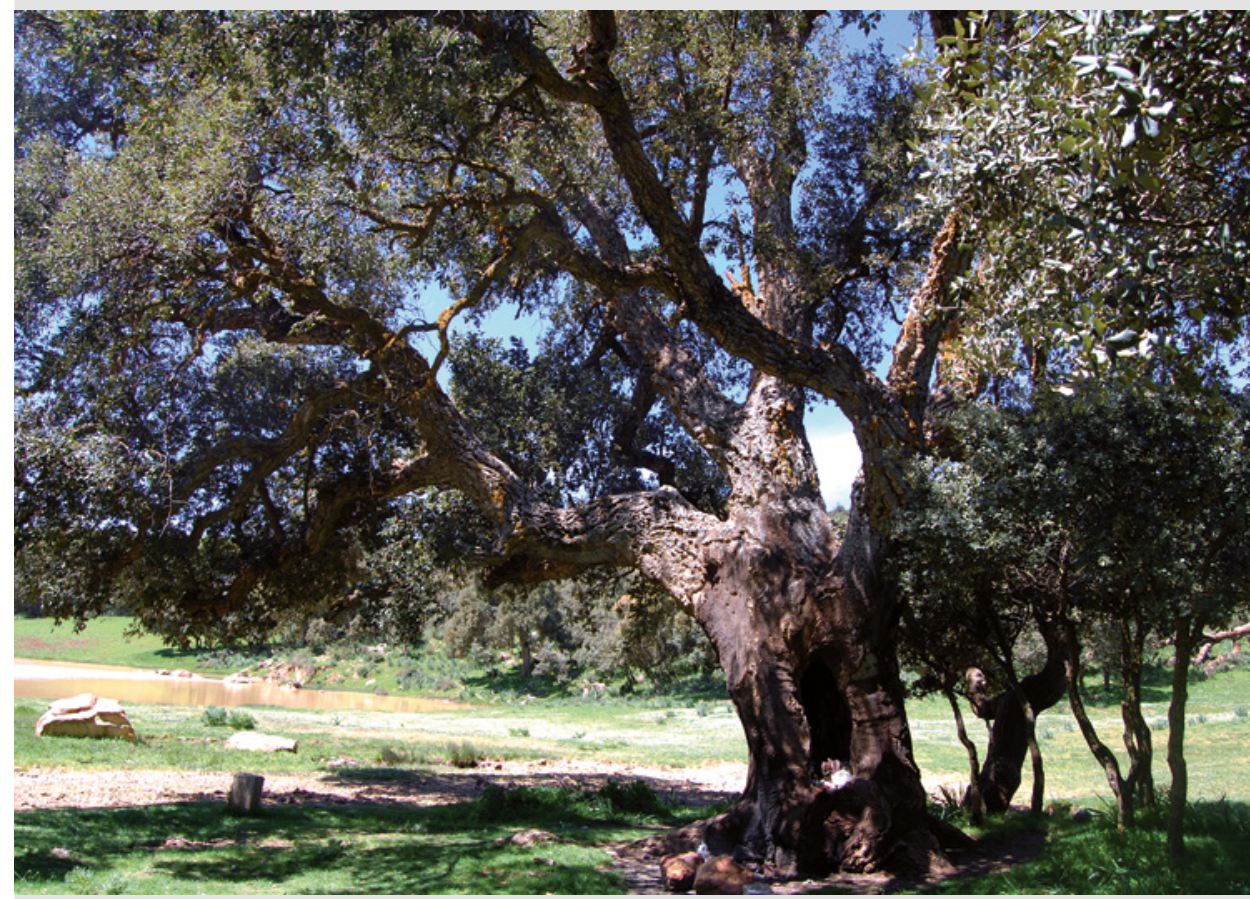

Photo 5.

Grosse tige de chêne-liège des basses altitudes.

Photo O. Naggar, 2013. topographique est très favorable pour $48 \%$ des peuplements. L'altitude varie entre 1290 et $1526 \mathrm{~m}$, mais la plupart des placettes (66\%) sont localisées entre 1390 et $1490 \mathrm{~m}$, ce qui classe le chêneliège de la zone dans l'étage de végétation supra-méditerranéen (Quézel, 2000). Le sol est généralement superficiel (52\% des placettes) et l'état de santé des peuplements est globalement satisfaisant $\left(\mathrm{IS}_{\text {moyen }}=1,58\right)$. Les peuplements présentent une structure générale irrégulière et sont composés d'arbres de différentes classes de grosseur avec un déficit accru de la classe des tiges de petite grosseur (figure 2a). Les hauteurs dominantes (figure $2 b$ ) sont bien représentées entre 6 et $8 \mathrm{~m}$ (40\%).

\section{Croissance du liège et production des arbres et des peuplements}

Les résultats du tableau III montrent qu'en moyenne le liège atteint l'épaisseur commerciale en 12 ans car le cumul moyen des largeurs des 11 premiers cernes complets est de 26,22 $\mathrm{mm}$. Cette valeur est peu variable entre les échantillons (CV = $22 \%)$. Un arbre peut produire $6,58 \mathrm{~kg}$ de liège sec par mètre carré de surface écorcée avec une densité de $230 \mathrm{~kg} / \mathrm{m}^{3}$. Durant une rotation (12 ans), la quantité de liège fournie par un arbre moyen est de $10,46 \mathrm{~kg}$, et celle d'un peuplement de densité exploitable moyenne de 260 pieds/ha est de $2791 \mathrm{~kg} / \mathrm{ha}$. Ces quantités de liège sont très variables entre les arbres et les placettes (CV > $50 \%$ ).

\section{Variables affectant la croissance en épaisseur du liège}

Les résultats de l'analyse du modèle obtenus pour prédire la croissance du liège montrent que l'épaisseur du liège est significativement plus affectée par les variables individuelles de l'arbre que par celles du milieu physique ou forestier (tableau IV). Parmi ces variables, nous citons l'état de santé et la grosseur des arbres qui 
Tableau III.

Caractérisation de la croissance du liège, de la production des arbres et des peuplements du chêne-liège en fin de cycle de production.

\begin{tabular}{|c|c|c|c|c|c|c|}
\hline & Code & Moyenne & Minimum & Maximum & Écart-type & $\begin{array}{c}\text { Coefficient de } \\
\text { variation (\%) }\end{array}$ \\
\hline 11 accroissements moyens annuels comp & & & & & & \\
\hline Épaisseur cumulée $(\mathrm{n}=125)(\mathrm{mm})$ & $\mathrm{E}_{11}$ & 26,22 & 10,37 & 42,68 & 5,71 & 21,79 \\
\hline Rendement du liège d'un arbre (kg) & $\mathrm{R}_{11}$ & 10,46 & 1,92 & 31,65 & 7,35 & 70,24 \\
\hline Production du peuplement (kg/ha) & $P_{11}$ & 2791,66 & 355,20 & 6148,40 & 1749,56 & 62,69 \\
\hline Poids du liège par unité de surface $(\mathrm{kg} / \mathrm{m})$ & Pds & 6,58 & 4,51 & 7,63 & 0,94 & 14,33 \\
\hline Poids volumique du liège $(\mathrm{kg} / \mathrm{m})$ & $\mathrm{p}$ & 230,40 & 162,65 & 274,60 & 35,62 & 15,40 \\
\hline
\end{tabular}

présentent une relation inverse avec la vitesse de croissance du liège. En fait, les meilleurs accroissements annuels du liège sont enregistrés chez les arbres sains ayant une faible grosseur. Par contre, les arbres affaiblis et de diamètres supérieurs produisent des cernes plus étroits. D'autres variables de second rang contribuent significativement à la variabilité de la croissance du liège. Il s'agit de la hauteur d'écorçage et de l'exposition de la placette. Elle augmente, mais faiblement, avec l'augmentation de la hauteur d'écorçage et en exposition favorable (sudouest). Même tous les coefficients de ces variables sont significatifs, les meilleures corrélations se manifestant entre la croissance du liège et l'état de santé de l'arbre $(r=-0,364)$ ou le diamètre $(r=-0,315)$. Bien que les coefficients obtenus dans le modèle soient très faibles et que les effets correspondants soient donc limités, nous pouvons dire que les deux premières variables (état de santé et grosseur du tronc) peuvent intervenir à elles seules pour déterminer la croissance du liège en fin de cycle, mieux que lorsqu'elle est déterminée en même temps par la hauteur d'écorçage et l'exposition du site. Pour le

\section{Tableau IV.}

Régression linéaire logistique pour prédire l'épaisseur du liège en fin de cycle de production.

Modèles basés sur 125 plaques échantillonnées.

\begin{tabular}{l|l|l|c|c|} 
Modèle & Variables & $\begin{array}{c}\text { Coefficient } \\
\pm \text { erreur standard }\end{array}$ & Z valeur & $\operatorname{Pr}(>|z|)$ \\
Facteur de & $\beta_{0}$ (intercept) & $-0,618 \pm 1,875$ & - & - \\
croissance & Déficit foliaire & $-0,091 \pm 0,021$ & $-4,202$ & $0,000^{\star \star *}$ \\
du liège & Diamètre de l'arbre & $-0,066 \pm 0,019$ & $-3,434$ & $0,000^{\star \star *}$ \\
& Hauteur écorçage & $3,093 \pm 1,875$ & 2,854 & $0,004^{\star *}$ \\
& Exposition & $0,957 \pm 0,411$ & 2,326 & $0,020^{\star}$
\end{tabular}

Coefficients du modèle \pm erreur standard ; $\beta_{0}$ : intercept ; valeur du test du rapport de vraisemblance ( $Z$ valeur); signification: *** $P<0,001 ;$ ** $P<0,01 ;$ * $P<0,05$. Performances du modèle : R-carré multiple $(0,341)$, AIC $(130,5)$, BIC $(150,3)$. reste des variables (P, Alt, PS, Nt), les résultats d'analyse ont montré un effet non significatif sur la croissance du liège mais aussi une indépendance linéaire (absence de toute multi-colinéarité entre les variables explicatives).

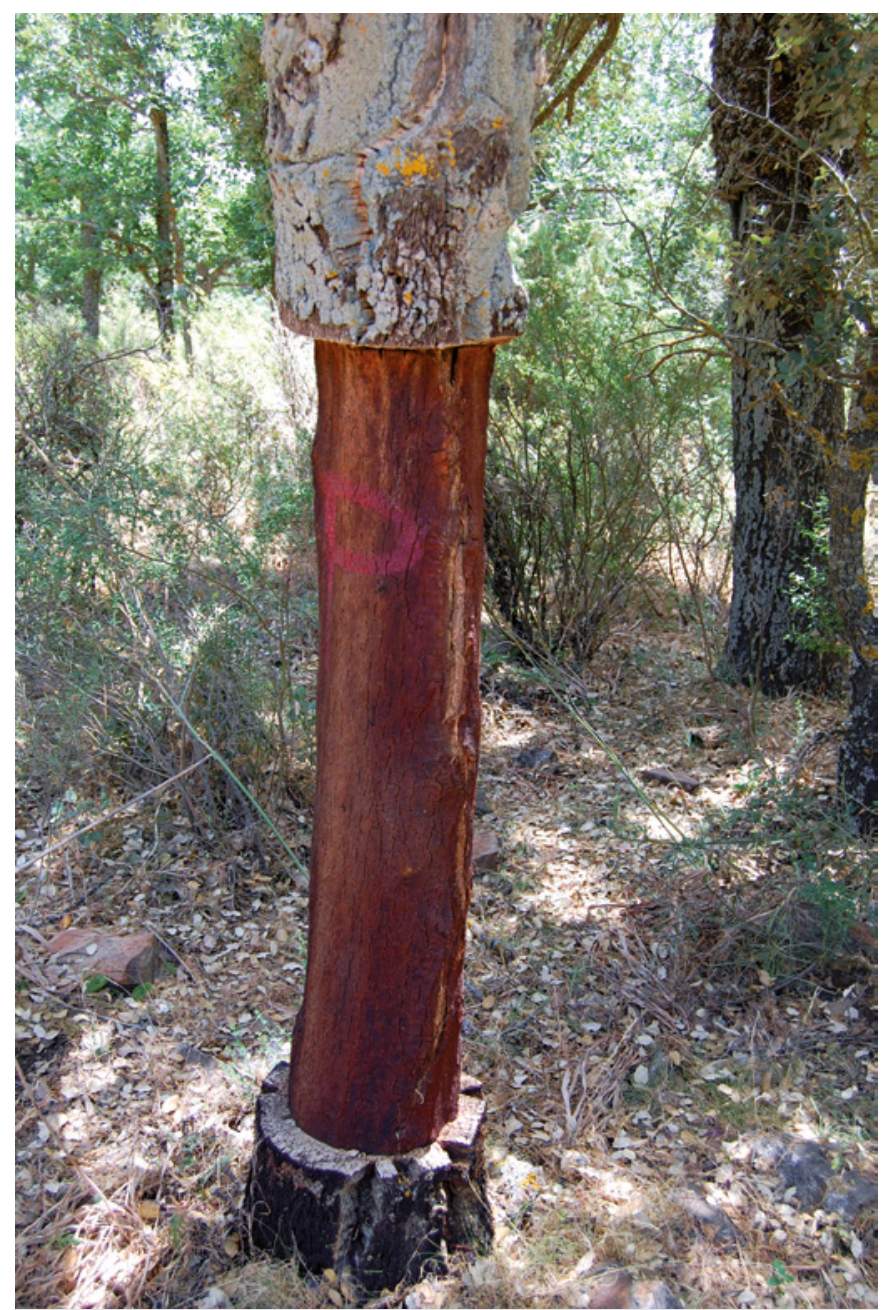

Photo 6.

Chêne-liège exploité illicitement en 2013.

Photo O. Naggar, 2013. 
Tableau V.

Modèle linéaire multiple pour prédire le rendement de l'arbre.

Modèle basé sur 634 arbres échantillonnés.

\begin{tabular}{l|l|r|r|c|} 
Modèle & Variables & $\begin{array}{c}\text { Coefficient } \\
\pm \text { erreur standard }\end{array}$ & t valeur & $\operatorname{Pr}(>|t|)$ \\
\hline Facteur de & $\beta_{0}$ (intercept) & $38,461 \pm 15,798$ & - & - \\
rendement & Hauteur totale & $2,746 \pm 0,198$ & 13,871 & $0,000^{\star \star \star}$ \\
\hline de l'arbre & Altitude & $-0,029 \pm 0,011$ & $-2,734$ & $0,00^{\star \star}$ \\
& Déficit foliaire & $-0,064 \pm 0,024$ & $-2,631$ & $0,008^{\star \star}$ \\
\hline
\end{tabular}

Coefficients du modèle \pm erreur standard ; $\beta_{0}$ : intercept ; valeur du test du rapport de vraisemblance ( $Z$ valeur ); signification: *** $P<0,001 ; * * P<0,01 ;{ }^{*} P<0,05$.

Performances du modèle : R-carré multiple $(0,297)$, R-carré ajusté $(0,289)$; test statistique $\left(\mathrm{F}_{\mathrm{obs}}: 37,79 ; 7,627 \mathrm{df}\right.$, $\mathrm{p}$-value $\left.<0,000\right)$.

(P, PS et Expo) et forestier (Nt) sont restées non significatives avec une présence d'une certaine colinéarité entre la densité du peuplement et l'altitude $(r=0,450)$.

\section{Variables affectant la production en liège des peuplements}

À l'échelle du peuplement, les résultats des analyses montrent une interaction significative entre la production de liège du peuplement et la hauteur dominante et, dans un degré moindre, avec l'état de santé général (tableau VI). Ceci explique que les peuplements les plus productifs sont aussi les plus hauts et en bonne santé. Dans le modèle, seule la hauteur dominante peut prévoir la production de

\section{Variables affectant le rendement en liège des arbres}

Les résultats de l'analyse des variables soumises au modèle montrent l'influence significative de certaines variables sur le rendement en liège des arbres (tableau $\mathrm{V}$ ). Parmi ces variables, nous trouvons la hauteur totale $(\mathrm{Ht})$ qui contribue très significativement dans l'estimation du rendement. Ceci explique que les arbres les plus hauts ont généralement un meilleur rendement. Le rendement des arbres est affecté aussi, mais avec un degré moindre, par leur état de santé et l'altitude. Ceci indique que les arbres vigoureux (Df faible) se développant en altitude moins élevée sont plus productifs que les arbres en très haute altitude. Enfin, le modèle considéré a montré que, malgré les faibles valeurs des coefficients des trois variables (mêmes toutes significatives) à effet réduit, la hauteur totale des arbres peut prévoir seule le rendement en liège car sa valeur de corrélation $(r=0,520)$ est proche de celle obtenue avec une régression multiple $(R=0,545)$. Les autres variables liées au milieu physique

\section{Tableau VI.}

Modèle linéaire multiple pour prédire la production $(\mathrm{kg} / \mathrm{ha})$ du peuplement des placettes $(n=53)$.

\begin{tabular}{|l|l|}
\hline Modèle & $\begin{array}{l}\text { Variables } \\
\pm \text { erreur standard }\end{array}$ \\
\hline $\begin{array}{l}\text { Facteur de } \\
\text { production } \\
\text { de l'arbre }\end{array}$ & $\begin{array}{l}\beta_{0} \text { (intercept) } \\
\text { Hauteur dominante } \\
\text { Indice de santé }\end{array}$ \\
\hline
\end{tabular}

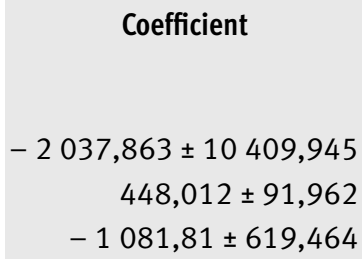

Coefficients du modèle \pm erreur standard; $\beta_{0}$ : intercept ; valeur du test du rapport de vraisemblance (Z valeur) ; signification : ${ }^{\star \star \star} P<0,001 ;{ }^{* \star} P<0,01 ;{ }^{*} P<0,05$. Performances du modèle : R-carré multiple $(0,430)$, R-carré ajusté $(0,356)$; test statistique $\left(\mathrm{F}_{\mathrm{obs}}: 5,789 ; 6 ; 46 \mathrm{df}, \mathrm{p}\right.$-value $\left.<0,000\right)$, AIC $(915,501)$, BIC $(921,412)$.
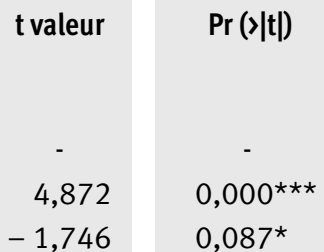

liège du peuplement avec une grande précision $(r=0,610$; $\mathrm{R}=0,656)$ sans recourir à la connaissance de son état sanitaire. Les autres variables (Expo, P, Alt et PS) n'ont montré aucune influence significative sur la variable réponse. En outre, les tests de multi-colinéarité ont montré que l'exposition et l'altitude ne sont pas indépendantes l'une de l'autre car il existe entre elles une forte corrélation $(r=0,816)$.

\section{Discussion}

\section{Facteurs influençant l'épaisseur du liège}

Le rythme de croissance du liège est lent $(2,38 \mathrm{~mm} /$ an) dans cette zone montagneuse. Ce rythme a été signalé dans d'autres suberaies montagneuses algériennes (Messaoudene et Metna, 2010 ; Dehane et Ghefar, 2017). En littoral ou en basse altitude, la vitesse de croissance du liège est par contre plus rapide et les accroissements sont plus larges (3 à $4 \mathrm{~mm} / \mathrm{an}$ en moyenne) (Pereira, 2007 ; Dehane et Ghefar, 2017). Une période de 12 ans est suffisante pour une grande partie du peuplement pour récolter des planches de liège bouchonnable $\left(R_{11}=27,73 \pm 1,35 \mathrm{~mm}\right.$; $\mathrm{CV}=22 \%)$. Cette première classe de qualité du liège est dominée par les épaisseurs standardisées (27-40 mm) au bon rendement industriel (57\%). Le reste des plaques de liège est plus mince $\left(R_{11}=\right.$ $24,75 \pm 1,75 \mathrm{~mm} ; \mathrm{CV}=$ $21,61 \%)$ et est alors destiné à la production de disques de liège, ou même à la trituration. 
L'épaisseur du liège semble être moins affectée par les facteurs du milieu. Ceci semble être dû à la détermination génétique, qui tend à maintenir ce type de croissance dans cette zone d'altitude indépendamment des conditions écologiques (Saccardy, 1937 ; Corona et al., 2005). Mais l'existence d'une catégorie d'arbres voisins évoluant dans des conditions identiques du site et fournissant des lièges de couches très variables (allant du mince à l'épais) est attribuée aux aptitudes individuelles de chaque arbre à réagir favorablement ou défavorablement aux facteurs liés au microsite (Saccardy, 1937 ; Natividade, 1956). Ce type de réponse des arbres est similaire à celui déjà signalé dans certaines forêts espagnoles (Torres et al., 1997 ; Sánchez-González et al., 2007) et italiennes (Corona et al., 2005). Nos résultats ont révélé que plusieurs facteurs pouvaient produire une variation de l'épaisseur du liège, en l'occurrence l'état sanitaire et le diamètre des arbres, mais aussi la hauteur d'écorçage de l'arbre et l'exposition du terrain. Ces résultats sont conformes aux travaux de Dehane et al. (2013b) qui ont observé que l'altération sanitaire des arbres réduit de près de $10 \%$ les accroissements annuels du liège. De plus, nos résultats ont montré que les jeunes arbres ont tendance à fournir une écorce légèrement plus épaisse que les arbres plus âgés. Ils sont en relation avec le cycle de vie de l'arbre où le déclin de l'épaisseur du liège commence à se manifester dès l'âge de 70 ans et devient plus prononcé après 120 ans (Natividade, 1956). Mais en pleine phase de production des arbres, de légères augmentations de l'épaisseur sont enregistrées avec le diamètre (Sánchez-González et al., 2007 ; Costa et al., 2003). Les expositions favorables aux pluies (sud-ouest) des sites peuvent améliorer la croissance du liège par

Photo 7.

Photo 8. rapport aux expositions plus sèches. Ces expositions ont une bonne disponibilité en eau (Nadezhdina et al., 2008 ; Ghalem et al., 2018). La densité du peuplement reste en revanche sans aucun effet sur la formation du liège, ce qui est conforme avec les travaux de Torres et al. (1997) et Sánchez-González et al. (2007).

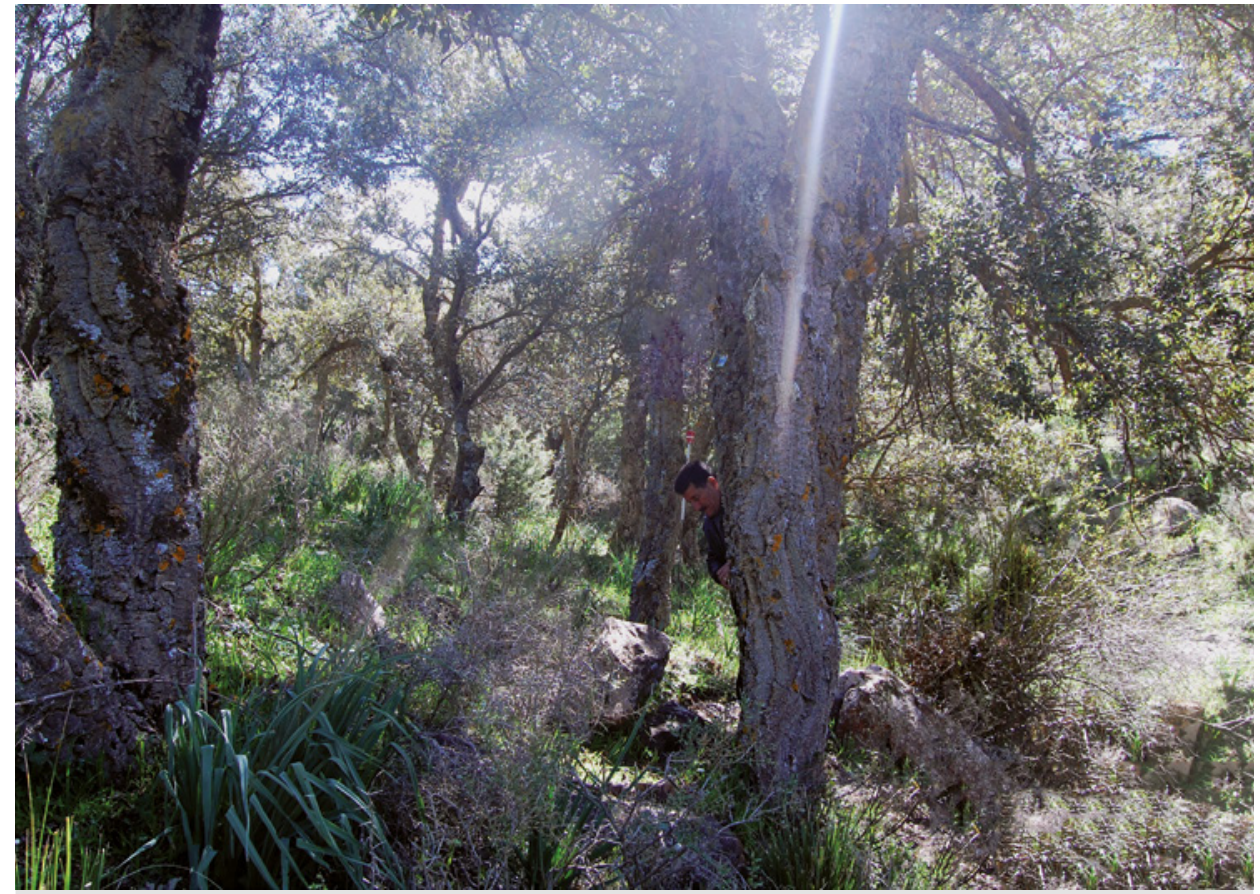

Suberaie pure des hautes altitudes (1500 m).

Photo O. Naggar, 2013.

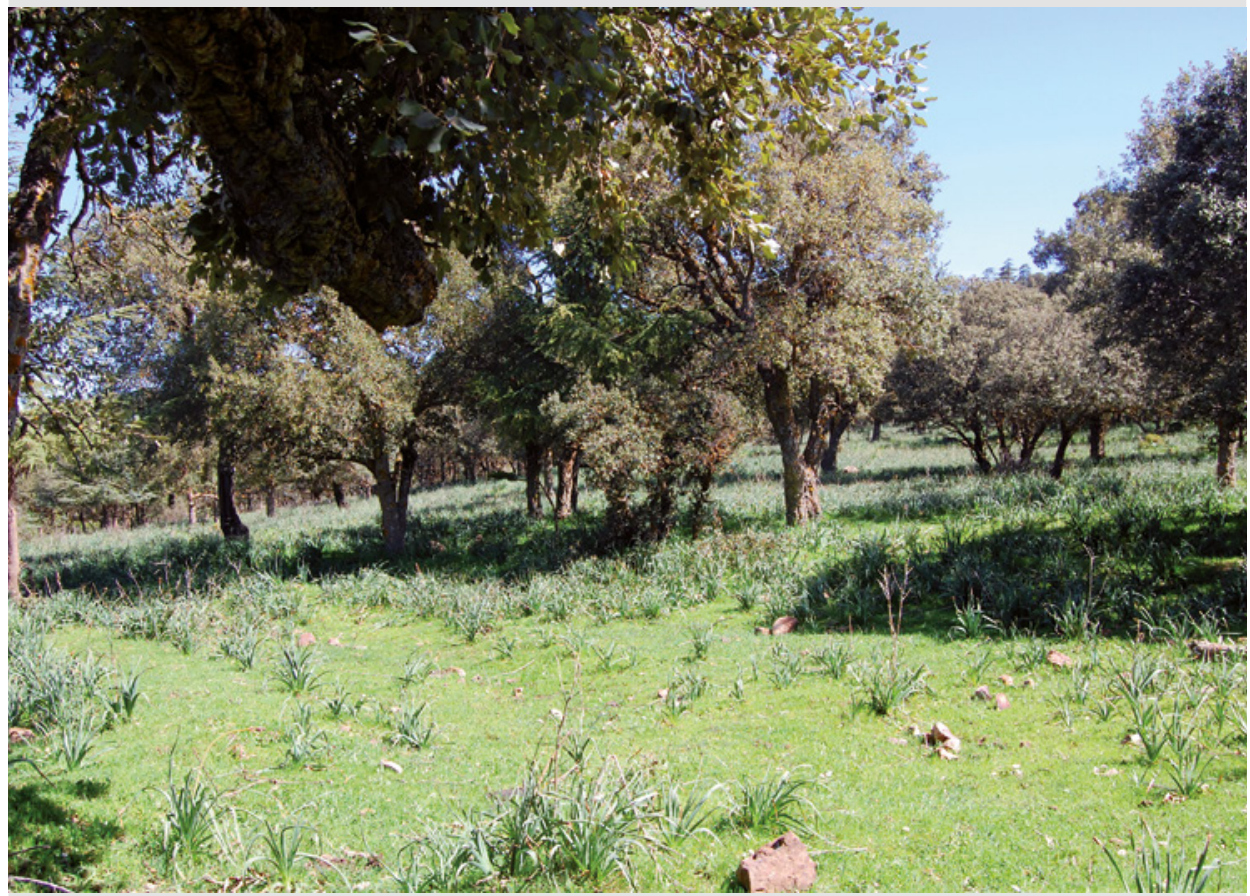

Suberaie pure à une altitude moyenne (1 $400 \mathrm{~m})$.

Photo O. Naggar, 2013. 


\section{Facteurs influençant le rendement des arbres en liège et la production des peuplements}

En forêt de Theniet El Had, l'analyse du modèle développé a permis de prédire que le rendement du liège est en relation avec la croissance des arbres exprimée par leur hauteur totale. En effet, les arbres les plus rentables sont généralement vigoureux, grands et hauts $(\mathrm{Ht}>12 \mathrm{~m}$, $D>0,40 \mathrm{~m}, \mathrm{R}_{11}=28,50 \mathrm{~kg}$ ). Ces arbres se développent en étage inférieur.

À l'échelle du peuplement, les résultats ont montré que la source de la variabilité de la production du liège réside essentiellement dans la qualité des sites indiquée par la croissance en hauteur dominante. En fait, en dépit de la faible densité enregistrée, la variabilité de production s'explique par une surdensité locale exercée sur les arbres.

À l'intérieur des peuplements, la croissance d'un arbre est largement conditionnée par la proximité et la dimension de ses voisins, et donc par l'espace vital disponible (Gaudin, 1997). Dans le cas de la suberaie de Theniet $\mathrm{El} \mathrm{Had}$, issue de régénération naturelle et non soumise aux conduites sylvicoles adéquates, la compétition locale entre arbres est accrue, engendrant des différences de croissance.

$\mathrm{Au}$ regard de nos résultats, les sites d'étude se regroupent en deux grands types de qualité.

Dans les sites favorables, les peuplements sont productifs, vigoureux et caractérisés par une bonne croissance des arbres ( $\mathrm{Hd}>10 \mathrm{~m} ; \mathrm{P}_{11}=2900 \mathrm{~kg} / \mathrm{ha}$ ). Ces peuplements sont localisés en étage inférieur, ce qui correspond à la limite altitudinale exigée par l'essence en Algérie (Saccardy, 1937). Ils croissent sur un sol à bonne disponibilité en eau (Nadezhdina et al., 2008 ; Paulo et al., 2015). En cette altitude, la production de liège semble suffisante mais elle reste toutefois inférieure à celle signalée par Saccardy (1937) sur le littoral algérien. Au Portugal, une telle production est atteinte avec la moitié seulement de la densité du peuplement (Natividade, 1956 ; Pereira, 2007).

Dans les sites non favorables, la production de liège est faible, de l'ordre de $1700 \mathrm{~kg} / \mathrm{ha}$, et les arbres ont une croissance ralentie $(\mathrm{Hd}<8 \mathrm{~m} ; \mathrm{D}=0,24 \mathrm{~m})$. Les peuplements se trouvent en altitude exceptionnelle (>1400 m), souvent sur des sols superficiels à faible capacité de rétention en eau (Richard, 1988 ; Nadezhdina et al., 2008 ; Paulo et al., 2015).

\section{Conclusion}

Dans le Parc national de Theniet El Had, le chêneliège présente une bonne adaptation au milieu de haute montagne. Il produit un liège à croissance lente, plus homogène, et d'une qualité très recherchée par l'industrie des bouchons. Les modèles développés nous ont permis d'acquérir les premières connaissances sur le mode de production du liège d'une suberaie naturelle non gérée. Les résultats montrent que la croissance en épaisseur du liège dépend surtout de l'aptitude individuelle des tiges. Les meilleurs accroissements sont enregistrés chez les jeunes individus, vigoureux, poussant dans les stations fraîches. Par ailleurs, la hauteur totale contribue à la variabilité du rendement des arbres. Ce dernier est aussi affecté, mais dans un degré moindre, par l'état de santé. De plus, à l'échelle des peuplements, la production de liège se montre fortement liée à la hauteur dominante. Cette dernière, incluse dans le modèle avec d'autres facteurs, nous permet de regrouper les sites d'étude en deux grands groupes de qualité à partir des facteurs qui ont une influence sur la production. Beaucoup d'autres qualités de site intermédiaires peuvent être dégagées, ce qui signifie que le terrain est très hétérogène et que les peuplements étudiés présentent une grande variabilité. Ces modèles devraient être améliorés dans le futur afin de mettre en évidence d'autres sources de variabilité encore non identifiées (au niveau du microsite, de l'arbre ou de la placette) de la production du liège. Ces modèles nous aident à proposer un plan de gestion durable et équilibré de la forêt basé sur la qualité des sites (bonne ou mauvaise production).

\section{Références bibliographiques}

Boudy P., 1950. Économie forestière nord-africaine. Tome 2 : Monographies et traitements des essences forestières. Paris, France, Larose, 878 p.

Boudy P., 1955. Économie forestière nord-africaine. Tome 4 : Description forestière de l'Algérie et de la Tunisie. Paris, France, Larose, 483 p.

Bouhraoua R. T., Villemant C., 2005. Mécanismes généraux de l'altération sanitaire des peuplements de chêne-liège de l'Algérie nord-occidentale. Protection in Oak Forests/Wet Palearctic Regional Section (IOBC/wprs) Bulletin, 28 (8) : 1-8.

Bouhraoua R. T., Piazzetta R., Berriah A., 2014. Les reboisements en chêne-liège en Algérie, entre contraintes écologiques et exigences techniques. Forêt Méditerranéenne, 35 (2) : 171-176.

Corona P., Dettori S., Filigheddu M. R., Maetzke F., Scotti R., 2005. Site quality evaluation by classification tree: an application to cork quality in Sardinia. European Journal of Forest Research, 124: 37-46. https://doi.org/10.1007/ s10342-004-0047-1

Costa A., Pereira H., Oliveira A., 2002. Influence of climate on the seasonality of radial growth of cork oak during a cork production cycle. Annals of Forest Science, 59: 429437. https://doi.org/10.1051/forest:2002017

Costa A., Pereira H., Oliveira A., 2003. Variability of radial 
growth in cork oak mature trees under cork production. Forest Ecology and Management, 175: 239-246. https:// doi.org/10.1016/s0378-1127(02)00145-7

Costa A., Barbosa I., Roussado C., Graça J., Spiecker H., 2016. Climate response of cork growth in Mediterranean oak (Quercus suber L.) woodlands of southwestern Portugal. Dendrochronologia, 38: 72-81. https://doi. org/10.1016/j.dendro.2016.03.007

Cuevas-Gozalo J. M., 1995. Ecuaciones de cubicación para el alcornoque del este Argelia. Forest Systems, 4 (1): 119-126. https://recyt.fecyt.es/index.php/IA/article/ view/4855

Dehane B., Bouhraoua R. T., Belhoucine L., Hamani F. Z., 2013a. La filière liège algérienne entre passé et présent. Forêt Méditerranéenne, 34 (2) : 143-152.

Dehane B., Bouhraoua R. T., Garcia de Ceca J. L., GonzalezAdrados J. R., 2013b. Effect of the health status on the cork production characteristics of Western Algeria cork oak stands. Forest Systems, 22 (1): 138-146. https://doi. org/10.5424/fs/2013221-02866

Dehane B., Ghefar M., 2017. Étude de la variabilité de croissance du liège dans le Nord Algérien. Geo-Eco-Trop, 41 (1) : 39-54.

DGF, 2013. Statistiques des incendies de forêts de chêneliège en Algérie, entre 1985 et 2012. Alger, Algérie, Direction générale des forêts.

DSF, 1991. Réseaux de surveillance de l'état sanitaire des forêts (réseau CEE et réseau Bleu) : Protocole pour les observations. DERF, Ministère de l'Agriculture et de la Forêt, $27 \mathrm{p}$.

Emberger L., 1955. Projet d'une classification biogéographique des climats. Annales de Biologie, 31 (56) : 249-455.

FAO, 2013. State of Mediterranean forests 2013. Rome, Italy, Food and Agriculture Organization of the United Nations, 189 p.

Ferreira A., Lopes F., Pereira H., 2000. Caractérisation de la croissance et de la qualité du liège dans une région de production. Annals of Forest Science, 57 (2) : 187-193. https://doi.org/10.1051/forest:2000169

Ghalem A., Barbosa I., Bouhraoua R. T., Costa A., 2018. Climate signal in cork-ring chronologies: A case study at Portugal (Benavente) and Algeria (HafirZarieffet). Tree-Ring Research, 74 (1): 15-27. https://doi. org/10.3959/1536-1098-74.1.15

Gaudin S., 1997. Quelques éléments d'écologie utiles au forestier. CFPPA/CFAA de Châteaufarine, BTSA gestion forestière, France, $88 \mathrm{p}$.

González-Adrados J. R., González-Hernandez R., Calvo-
Haro R., 2000. La predicción del calibre de corcho al final del turno y su aplicación al muestreo de la producción. Forest Systems, 9 : 363-373. https://recyt.fecyt.es/index. $\mathrm{php/IA/article/view/2629}$

Imdadullah M., Aslam M., Altaf S., 2016. mctest: An R Package for Detection of Collinearity among Regressors. The R Journal, 8 (2): 499-509. https://journal.r-project. org/archive/2016/RJ-2016-062/index.html

Lamey A., [1893] 2016. Le chêne-liège, sa culture et son exploitation. [Berger-Levrault]. Paris, France, Hachette Bnf, 308 p.

Masson G., 2005. Autécologie des essences forestières. Comment installer chaque essence à sa place. Tome 1 . Paris, France, Tec et Doc Lavoisier, 306 p.

Mendes M. P., Ribeiro L., David T. S., Costa A., 2016. How dependent are cork oak (Quercus suber L.) woodlands on groundwater? A case study in southwestern Portugal. Forest Ecology and Management, 378: 122-130. https:// doi.org/10.1016/j.foreco.2016.07.024

Messaoudene M., Metna B., 2010. Étude de la qualité du liège des subéraies orientales de Tizi-Ouzou. Protection in Oak Forests/Wet Palearctic Regional Section (IOBC/ wprs) Bulletin, $57: 33-40$.

Montero G., Torres E., Cañellas I., Ortega C., 1996. Modelos para la estimación de la producción de corcho en alcornocales. Forest Systems, 5 (1): 197-127. https:// recyt.fecyt.es/index.php/IA/article/view/4923

Nadezhdina N., Ferreira M. I., Silva R., Pacheco C. A., 2008. Seasonal variation of water uptake of a Quercus suber tree in Central Portugal. Plant Soil, 305: 105-119. https://doi.org/10.1007/s11104-007-9398-y

Natividade J. V., 1956. Subériculture. Nancy, France, Éditions de l'École Nationale des Eaux et Forêts, 303 p.

Paulo J. A., Tomé M., 2010. Optimization of cork debarking rotation for sites of different cork productivity and quality. In: Parrota J. A., Carr M. A. (eds). Proceedings of the XXIII IUFRO World Congress: Forests for the future - Sustaining Society and the environment, Seoul (Korea), 23-28 August 2010, International forestry review, 12 (5): 40.

Paulo J. A., Tomé J., Tomé M., 2011. Non linear fixed and random generalized height-diameter models for Portuguese cork oak stands. Annals of Forest Science, 68: 295-309. https://doi.org/10.1007/s13595-011-0041-y . 
extractions using cork growth index modelling. Agroforest Systems, 91 (2): 221-237. https://doi.org/10.1007/ s10457-016-9922-2

Paulo J. A., Tomé M., 2017. Using the SUBER model for assessing the impact of cork debarking rotation on equivalent annual annuity in Portuguese stands. Forest Systems, 26 (1): 1-11. https://doi.org/10.5424/ fs/2017261-09931

Pereira H., 2007. Cork: Biology, production and uses. Amsterdam, Netherlands, Elsevier, 346 p. https://doi. org/10.1515/hfsg.1996.50.2.111

Pereira H., Lopes F., Graça J., 1996. The evaluation of the quality of cork planks by image analysis. Holzforschung, 50: 111-115.

PNTH, 2006. Atlas des parcs nationaux algériens. Alger, Algérie, ED-DIWAN, 98 p.

Quézel P., 2000. Réflexions sur l'évolution de la flore et de la végétation au Maghreb méditerranéen. Paris, France, Ibis Press, 117 p.

R Core Team, 2018. R: A Language and Environment for Statistical Computing. Austria, R Foundation for Statistical Computing. https://www.R-project.org

Richard P., 1988. La croissance du chêne-liège. Forêt Méditerranéenne, 10 (1) : 169-171.

Rondeux J., 1999. La mesure des arbres et des peuplements forestiers. Gembloux, Belgique, Les Presses agronomiques de Gembloux, 251 p.

Saccardy L., 1937. Notes sur le chêne-liège et le liège en Algérie. Bulletin de la station de recherche forestière du Nord de l'Afrique, T. II, fasc. 3, septembre 1937, 271-374. https://doi.org/10.3406/jatba.1938.5885

Sánchez-González M., Tomé M., Montero G., 2005. Modelling height and diameter growth of dominant cork oak trees in Spain. Annals of Forest Science, 62: 633-643. https://doi.org/10.1051/forest:2005065

Sánchez-González M., Calama R., Cañellas I., Montero G., 2007. Variables influencing cork thickness in Spanish cork oak forests: A modelling approach. Annals of Forest Science, 64: 301-312. https://doi.org/10.1051/ forest:2007007

Sánchez-González M., Stiti B., Chaar H., Cañellas I., 2010. Dynamic dominant height growth model for Spanish and Tunisian cork oak (Quercus suber L.) forests. Forest Systems, 19 (3): 285-298. https://doi.org/10.5424/ fs/2010193-8495

Torres E., Montero G., Suarez M. A., 1997. Relación entre la densidad de la masa y la producción de corcho en montes alcornocales del sur de España. In: Puertas F., Rivas M. (eds). II Congreso Forestal Nacional, Tomo IV,

\section{9-534. https://doi.org/10.2307/i.ctvn96g1f.20}

Vázquez-Piqué J., Pereira H., 2005. Mixed models to estimate tree oven-dried cork weight in Central and Southern Portugal. Forest Ecology and Management, 213 (1): 117-132. https://doi.org/10.1016/i. foreco.2005.03.047

Zine M., 1992. Situation et perspectives d'avenir du liège en Algérie. Actes du colloque "Les Suberaies méditerranéennes », Institut méditerranéen du liège, France.

\begin{tabular}{|c|c|}
\hline \multicolumn{2}{|c|}{ Naggar et al. - Contribution des auteurs } \\
\hline RÔLE DU CONTRIBUTEUR & NOMS DES AUTEURS \\
\hline Conceptualisation & $\begin{array}{l}\text { T. R. Bouhraoua, O. Naggar, } \\
\text { E. Torres }\end{array}$ \\
\hline Gestion des données & T. R. Bouhraoua, O. Naggar \\
\hline Analyse formelle & $\begin{array}{l}\text { T. R. Bouhraoua, O. Naggar, } \\
\text { E. Torres }\end{array}$ \\
\hline $\begin{array}{l}\text { Acquisition du } \\
\text { financement }\end{array}$ & Pas de soutien financier \\
\hline Enquête et investigation & O. Naggar, M. Zedek \\
\hline Méthodologie & T. R. Bouhraoua, O. Naggar \\
\hline Gestion de projet & T. R. Bouhraoua \\
\hline Ressources & $\begin{array}{l}\text { T. R. Bouhraoua, O. Naggar, } \\
\text { M. Zedek }\end{array}$ \\
\hline Logiciels & $\begin{array}{l}\text { T. R. Bouhraoua, O. Naggar, } \\
\text { E. Torres }\end{array}$ \\
\hline Supervision & T. R. Bouhraoua \\
\hline Validation & $\begin{array}{l}\text { T. R. Bouhraoua, O. Naggar, } \\
\text { M. Zedek }\end{array}$ \\
\hline Visualisation & T. R. Bouhraoua, O. Naggar \\
\hline $\begin{array}{l}\text { Écriture - Préparation } \\
\text { de l'ébauche originale }\end{array}$ & T. R. Bouhraoua, O. Naggar \\
\hline $\begin{array}{l}\text { Écriture - Révision } \\
\text { et édition }\end{array}$ & $\begin{array}{l}\text { T. R. Bouhraoua, O. Naggar, } \\
\text { M. Zedek, E. Torres }\end{array}$ \\
\hline
\end{tabular}

Bois et Forêts des Tropiques - Revue scientifique du Cirad

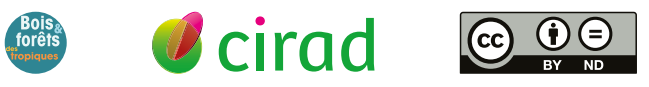

Cirad - Campus international de Baillarguet, 34398 Montpellier Cedex 5, France - Contact : bft@cirad.fr - ISSN : L-0006-579X 